\title{
Inteligencias Múltiples, Creatividad y Lateralidad, nuevos retos en metodologías docentes enfocadas a la innovación educativa
}

\author{
Yésica Casado Aragoneses, Fátima Llamas Salguero y Verónica López Fernández. \\ Universidad Internacional de la Rioja
}

Recepción: 20 de octubre de 2015 | Revisión: 20 de octubre de 2015 | Aceptación/Publicación: 21 de octubre de 2015

Correspondencia: fatima.llamas@unir.net

Citar: Casado, Y., Llamas-Salguero, F. y López-Fernández, V. (2015). Inteligencias Múltiples, Creatividad y Lateralidad, nuevos retos en metodologías docentes enfocadas a la innovación educativa. ReiDoCrea, 4, 343-358.

\begin{abstract}
Resumen: El artículo se centra en el desarrollo de la creatividad, el trabajo con las Inteligencias Múltiples y la lateralidad. Recoge la información más destacada de estos conceptos y el análisis de una serie de pruebas de Inteligencias Múltiples y de Lateralidad a un grupo de 30 alumnos de $2^{\circ}$ de Educación Primaria. Entre los resultados podemos destacar que existen niveles más bajos en la Inteligencia Lingüística, Viso-espacial y Cinestésico-corporal. Asimismo, se han encontrado niños con problemas de lateralidad, por cruces o por falta de establecimiento de la misma. Por todo ello se indican procedimiento y pautas para enfocar a nuevas metodologías docentes para el desarrollo de dichas inteligencias y la lateralidad, siempre teniendo como fin último el desarrollo de la creatividad. Para contrastar dicho fin se han valorado los niveles de creatividad de estos niños (grupo experimental) y se han contrastado con los de otro grupo equivalente (grupo control), encontrando diferencias significativas entre ambos. Por ello, se puede concluir que el desarrollo de estas variables relacionadas en las Inteligencias Viso-espacial, Cinestésicocorporal, Lingüística y lateralidad son adecuados para el desarrollo de la creatividad.
\end{abstract}

Palabras Clave: Creatividad | Inteligencia | Lateralidad cerebral

Multiple Intelligences, Creativity, and Lateral Dominance, New Challenges in Teaching Methodologies Focused on Educational Innovation

\begin{abstract}
The article focuses on the development of creativity, work with multiple intelligences and laterality. Collect the most important information of these concepts and analysis of a series of tests of Multiple Intelligences and laterality a group of 30 students of 2 nd of Primary Education. Among the results we emphasize that there are lower levels in linguistic intelligence, visual-spatial and bodily-Kinesthetic. It also found children with laterality, by crosses or lack of establishing it. Therefore procedures and guidelines to focus on new teaching methodologies for the development of these intelligences and laterality are indicated, always with the ultimate aim to develop creativity. To test this purpose are rated levels of creativity of these children (experimental group) and have been compared with those of an equivalent group (control group), and found significant differences. Therefore, one can conclude that the development of these related variables in the visual-spatial Intelligence, Bodily-Kinesthetic, Linguistic and laterality are suitable for the development of creativity.
\end{abstract}

Keywords: Creativity | Intelligence | Lateral Dominance

\section{Introducción}

En los últimos años, la educación va encaminada a la formación integral de los niños, por lo que se tienen que abordar desde las aulas distintas áreas, metodologías, formas de presentar la información, etc. Para poder lograrlo, es necesaria que la formación de los docentes en estos campos también sea tenida en cuenta y llevada a cabo en las mejores condiciones. Sin embargo, en muchas ocasiones, hay temáticas que pasan desapercibidas entre los docentes. Por ello, con este artículo, se pretende ahondar un poco en tres elementos claves para poder conseguir esa educación integral tan deseada, como son la creatividad, la lateralidad y las inteligencias múltiples.

Partimos de la base de que para que se pueda llevar a cabo un correcto aprendizaje, es necesario que estén bien establecidos y desarrollados ciertos elementos y sistemas de nuestro organismo, de modo que el aprendizaje pueda ser pleno y nuestro cuerpo esté al servicio del mismo, centrándonos en los aspectos más superiores. Es decir, para que el aprendizaje se pueda llevar a cabo, es necesario que nuestro sistema visual se encuentre bien desarrollado, así como el auditivo. También es trascendente que estos dos sentidos, junto con los demás y el resto de receptores del organismo, 
capten bien la información y la integren de manera adecuada, pudiendo dar respuesta a las necesidades que plantea el entorno. Por otro lado, es necesario que la lateralidad esté bien establecida, gracias a la inhibición de una serie de reflejos primitivos y el desarrollo correcto de los patrones motrices básicos, habilidades motrices, etc. Para que todo esto tenga un efecto positivo en el rendimiento del día a día, es preciso que el cuerpo calloso esté bien activado, permitiendo una correcta relación entre ambos hemisferios.

Por otro lado, y ya centrándonos en un nivel más abstracto y no tan orgánico, podemos destacar la necesidad de trabajar con los alumnos aspectos tales como la memoria, la creatividad, la organización espacial, la atención, etc. En esta investigación se abordan elementos como la creatividad, las inteligencias múltiples y la lateralidad, incluyendo elementos para el trabajo con y de las mismas, favoreciendo el máximo desarrollo de los alumnos.

La creatividad y las Inteligencias Múltiples son elementos que a día de hoy están creciendo en popularidad y en aplicabilidad en las aulas, principalmente las Inteligencias Múltiples como metodología para la enseñanza de nuevos conocimientos. Su inclusión supone un gran avance en la educación, dando cabida a todos los niños, sean cuales sean sus capacidades predominantes. Esto resulta de gran importancia y trascendencia, ya que lo que se busca desde las aulas es que todos los niños estén implicados, y consigan el máximo desarrollo de sus capacidades. Asimismo, la creatividad es un elemento fundamental a trabajar en el aula ya que debe ser fomentada desde edades tempranas.

Por otro lado, la lateralidad tiene que esta esté bien establecida para que el aprendizaje del niño sea fructífero.

La importancia de este estudio recae en valorar los niveles, como grupo-clase, de las distintas inteligencias múltiples, obteniendo aquellas en las que los niveles están más bajos. Asimismo, se valora la lateralidad, viendo si esta está bien establecida o no, y comprobando si existe algún tipo de vinculación con los datos anteriormente registrados en las inteligencias múltiples.

A tal efecto, cabe destacar investigaciones como las de Pérez (2009), según el cual la creatividad va decreciendo con el paso de los años, y en muchas ocasiones el sistema educativo contribuye a ello, ya que hace que se hagan rígidos e inflexibles, aspecto que apoya también Bernal (2003). Asimismo, el trabajo con inteligencias múltiples de Gardner $(1983,1993)$ está teniendo efectos muy positivos en el desarrollo de los niños en el ámbito educativo. Por otro lado, Rius (1989) y Ferré y Aribau (2002) apuntan la necesidad de tener en cuenta en los centros escolar la lateralidad, así como la evaluación de la misma. Este estudio trata de sumarse a los anteriores y de aportar nuevas actividades para trabajar estos elementos en el aula.

\section{Creatividad}

La creatividad está sufriendo, en los últimos años, un aumento de su popularidad y trascendencia en la sociedad, así como dentro de las aulas, pues cada vez se toma más consciencia de la necesidad de que esta sea trabajada, como defiende De Bono (1994). En sus inicios, donde podemos destacar a autores como Poincaré (1913), Wallas (1926), Guildford (1967) y Torrance (1962, 1974), entre otros muchos, no se concebía tan más, siendo fruto de investigación, introduciéndose en las clases, etc., pues se ha visto la necesidad de que esta sea trabajada según De Bono (1994). 
Asimismo, y como destaca Robinson (2009), es importante que la creatividad forme parte del sistema educativo, por las repercusiones positivas que conlleva.

El concepto de creatividad ha sido abordado a lo largo de la historia por muchas personas, por lo que las definiciones relativas a este concepto son numerosas. Entre ellas, podemos encontrar las siguientes (Esquivias, 2004, p.4-5): "Según Thurstone (1952) es un proceso para formar ideas o hipótesis, verificarlas y comunicar los resultados, suponiendo que el producto creado sea algo nuevo". Según Sillamy (1973) es la disposición para crear que existe en estado potencial en todo individuo y en todas las edades".

Gardner (1993), autor del que trataremos más adelante por su vinculación directa con las Inteligencias Múltiples, defiende que una persona puede ser creativa en un campo determinado, pero no necesariamente en todos. Asimismo, la persona creativa sería aquella que elabora productos nuevos y aceptados, siendo capaz de resolver los problemas de manera regular.

\begin{tabular}{|c|c|}
\hline AUTOR & PRINCIPALES APORTACIONES \\
\hline $\begin{array}{l}\text { WALLAS } \\
(1926)\end{array}$ & 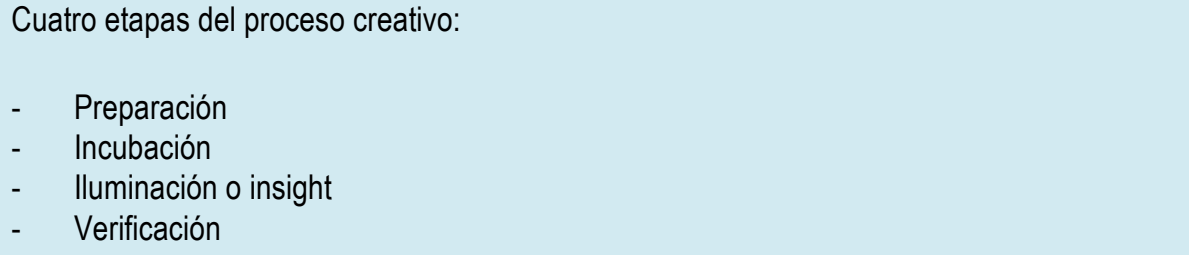 \\
\hline $\begin{array}{l}\text { GUILFORD } \\
\text { (1951) }\end{array}$ & $\begin{array}{l}\text { - Pensamiento divergente: pensamiento creativo. Se caracteriza por: fluidez, flexibilidad, } \\
\text { originalidad y elaboración. } \\
\text { - } \quad \text { Pensamiento convergente: razón, lógica y patrones fijos. }\end{array}$ \\
\hline $\begin{array}{l}\text { AMABILE } \\
(1983)\end{array}$ & $\begin{array}{ll}\text { - } & \text { Cinco pasos para llegar al producto deseado (Ver Figura 1). } \\
\text { - } & \text { Influencia del contexto, la motivación, la evaluación y las recompensas. }\end{array}$ \\
\hline $\begin{array}{l}\text { DE BONO } \\
(1994)\end{array}$ & $\begin{array}{ll}\text { - } & \text { Pensamiento lateral: relacionado con la creatividad } \\
\text { - } & \text { Pensamiento vertical: es analítico, selectivo y no se sale del camino pautado. }\end{array}$ \\
\hline
\end{tabular}

Dado que la creatividad es campo muy amplio y extenso y, según lo que hemos ido comentando de diferentes autores, las aportaciones son muy variadas, se ha optado por que, una de las maneras más coherentes para englobar todos los aspectos de la misma, es tener en cuenta varias dimensiones:

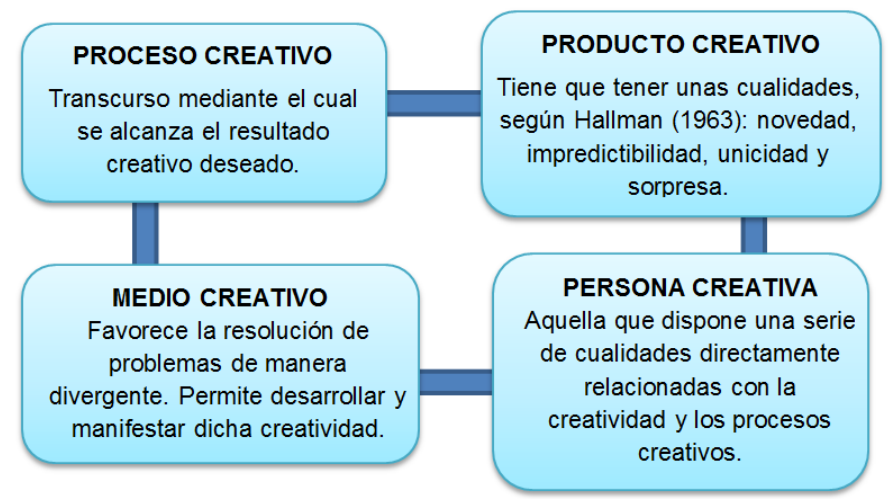

Figura 2. Componentes de la creatividad 
Respecto a las características de las personas creativas, estas también son numerosas, y es muy difícil determinar todas ellas, así como que una única persona las cumpla todas. Por ello, podemos destacar algunas de ellas, siguiendo lo recogido y aportado por Mackinnon (1980): son inteligentes, con una fuerte motivación intrínseca y gran seguridad y confianza en ellos mismos. Buscan la verdad, belleza, lo complejo y asimétrico. Asimismo, presentan una gran flexibilidad mental.

Al igual que ocurre en otros campos, este tampoco está exento de creencias y mitos (Ver Figura 3) que son erróneos, popularmente aceptados y extendidos. Estos son recogidos, entre otros, por De Bono (1994), teniendo nosotros, como docentes o profesionales, que cambiar estos puntos de vista:

\section{MITOS CREATIVIDAD}

La creatividad es un talento natural, no se enseña. Sin embargo, está si tiene que ser trabajada, y mediante diversas técnicas.

Solo con la intuición se puede ser creativo.

Solo los artistas con creativos, o las personas rebeldes. Sin embargo, esta abarca numerosos campos y ámbitos.

Inteligencia y creatividad son lo mismo. Realmente no son lo mismo, aunque están estrechamente relacionadas.

Figura 3. Mitos de la creatividad

\section{Inteligencias múltiples}

El concepto de inteligencia ha evolucionado mucho a lo largo de los años, desembocando en un estado actual en el que se considera la existencia de diversas inteligencias, ocho, según recoge Gardner (1983, 1993). Tradicionalmente se ha considerado la inteligencia como una sola, siendo esta de una mayor o menor capacidad y no pudiéndose modificar. Sin embargo, los avances neuropsicológicos han permitido cambiar esta idea y aceptar nuevas concepciones, lo cual ha tenido y está teniendo muchas repercusiones, sobre todo a nivel académico.

Un pionero en los estudios de la inteligencia fue Binet (1894), dentro de los modelos de la inteligencia monolítica, el cual propuso la valoración de la misma a través de habilidades comprensivas, de capacidades aritméticas, razonamiento, vocabulario, etc. Parte de la idea de que una materialización de la inteligencia, es la velocidad con la que se produce el aprendizaje. Asimismo, introdujo el concepto de Edad Mental y Cociente Intelectual y dio lugar a la creación de la Escala Binet-Simon (1905). Más adelante, Spearman (1923), desarrolló el modelo de la inteligencia factorial o factor $\mathrm{g}$ de Spearman, de modo que la inteligencia estaba compuesta por dos factores, uno de ellos más general y denominado $G$ (sería el elemento heredado) y una serie de factores específicos, conocidos como factores $\mathrm{s}$, que incluye los elementos verbales, numérico, espacial, entre otros.

También Guilford $(1965,1986)$ creó la Teoría de la Estructura del Intelecto, desarrollando un modelo cúbico de inteligencia, constituido por 150 factores que influían en la misma. 
Destacamos además a Piaget (1969) que resaltaba la importancia de la adaptación al medio circundante, por lo que acuñó los términos de asimilación (forma de enfrentarse a los estímulos del entorno) y acomodación (modificación de los esquemas anteriores para poder dar respuesta a las demandas del medio). Por otra parte Stenberg (1985) desarrolló la Teoría Triárquica de la Inteligencia, según la cual dentro de la inteligencia podríamos distinguir una más analítica, la cual nos permite adquirir y almacenar la información, así como establecer relaciones entre los diferentes elementos que forman parte de un problema. Otra inteligencia sería la sintética o creativa, que codifica y compara la información, con la intuición y creatividad, pudiendo extrapolarlo a diversas situaciones. Finalmente, concibe una inteligencia práctica, permitiendo adaptarnos al mundo real y resolver los problemas cotidianos de manera exitosa.

Como podemos observar, son diversas las teorías existentes al respecto, y múltiples los enfoques que se le está dando. Por ello, y dado que es una de las teorías más utilizadas actualmente, tanto a nivel teórico como práctico, nos vamos a centrar en la Teoría de las Inteligencias Múltiples de Gardner (1983, 1993). Esta surge de la concepción de que los aspectos de la inteligencia humana no pueden ser explicados desde una perspectiva monolítica o factorial, como anteriormente se ha expuesto, sino que el éxito o la acción intelectual de las personas tiene que ser explicado desde diferentes perspectivas. "Dado que los test de $\mathrm{Cl}$ tienen limitaciones y no evalúan todas las habilidades que poseemos y utilizamos, el doctor Gardner [...] a la conclusión de que no existe una sola manera de ser inteligente, sino muchas maneras distintas". (Armstrong, 2008, p.18-19). Es decir, Gardner (1993) defiende que hay una visión pluralista de la mente, dentro de la cual podemos reconocer varias facetas cognitivas, dando lugar a diferentes estilos y potenciales cognitivos.

"La competencia cognitiva del hombre queda mejor descrita en términos de un conjunto de habilidades, talentos o capacidades mentales, que denominamos "inteligencias". Todos los individuos normales poseen cada una de estas capacidades en un cierto grado; los individuos difieren en el grado de capacidades y en la naturaleza de la combinación de estas capacidades." (Gardner, 1995, p. 37).

De este modo Gardner presenta las distintas inteligencias, siendo siete inicialmente y añadiendo con posterioridad la naturalista. Las ocho inteligencias de Gardner son: Inteligencia Lingüística, Inteligencia Lógico-matemática, Inteligencia Viso-espacial, Inteligencia Musical, Inteligencia Corporal-cinestésica, Inteligencia Interpersonal, Inteligencia Intrapersonal e Inteligencia Naturalista. Según Amarís (2002), cada una de estas inteligencias posee unas capacidades y procedimientos propios, manifestándose a través de una serie de características específicas en cada una de ellas.

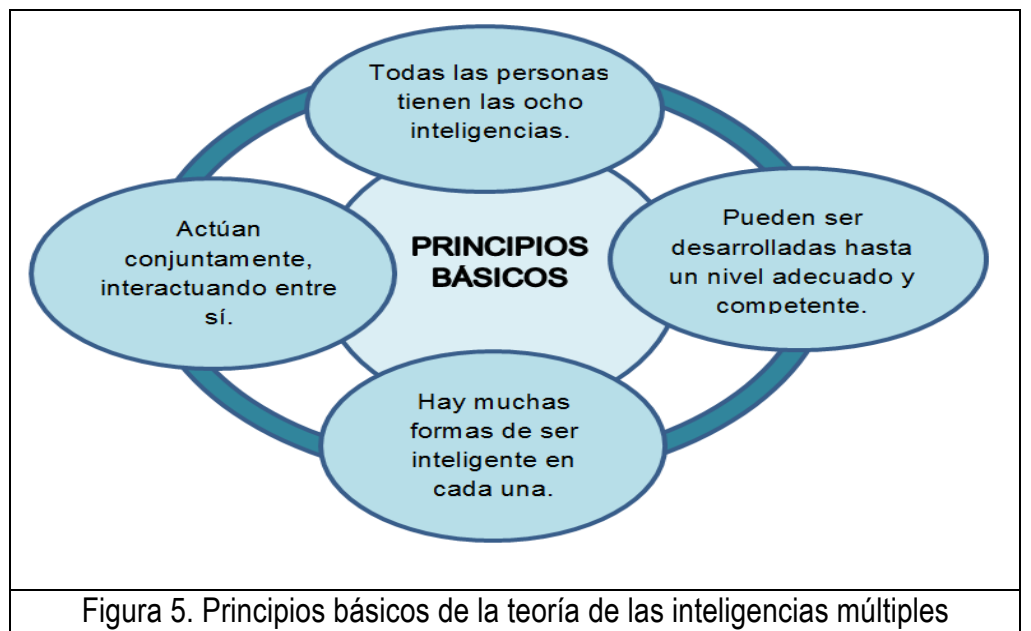




\section{Lateralidad}

La lateralidad, por su influencia en el aprendizaje y rendimiento escolar, es un aspecto cada vez más estudiado, aunque no siempre se tiene en consideración, por desconocimiento de la misma por parte de algunos docentes, lo cual debería ser modificado. Asimismo, hay que destacar la importancia que tienen todos los elementos corporales y espaciales en el desarrollo de la lateralidad, como recoge Rigal (2006).

Son numerosas las definiciones que se han planteado al respecto, entre las que podemos destacar la de Le Boulch (1981), según el cual, la lateralidad es la manifestación y expresión de una predominancia motriz, en relación con las partes del cuerpo y la integración de sus mitades derecha e izquierda. Este predominio está relacionado con la aceleración del proceso madurativo de los centros sensoriomotrices en alguno de los dos hemisferios.

Según Dorsch (1985), sería la dominancia lateral en la estructura y función de los órganos que tenemos duplicados; es decir, ojos, oídos, brazos, piernas, etc., pero principalmente en la mano.

Desrosiers (2005) apunta que la lateralidad se asocia a la capacidad de discriminación de derecha e izquierda, así como la capacidad de usar de manera dominante uno de los segmento.

Posteriormente, Rigal (2006), dice que la lateralidad sería el predominio en la función de una de las dos partes que son simétricas de nuestro organismo. Podemos encontrar varias clasificaciones o terminologías para referirnos a los tipos de lateralidad, nos vamos a centrar en los más usados, recogidos por Jiménez, Velázquez y Jiménez (2003):

- Diestro: utiliza su parte derecha del cuerpo para llevar a cabo las diferentes acciones, gracias a un dominio cerebral izquierdo.

- Zurdo: en la realización de las diferentes actividades utiliza su lado izquierdo, debido a una dominancia cerebral derecha.

- Zurdería contrariada: su dominancia corporal sería izquierda, pero dadas una serie de circunstancias sociales o culturales, utiliza el lado derecho.

- Ambidextrismo: es la utilización de los dos lados del cuerpo de manera indistinta, pero es muy complicado encontrarlo en estado puro, por lo que suele ocasionar problemas. Al no estar clara la dominancia, se usa la derecha e izquierda de manera indistinta.

- Lateralidad cruzada: dominancia de unos segmentos corporales sobre otros, siendo estos diestros o zurdos, pero al contrario en otros segmentos. También puede haber cruces, si la lateralidad es toda zurda, pero diestra, por ejemplo, en el ojo, sería zurdo con cruce visual.

- Lateralidad sin definir: se da cuando no se detecta un patrón de utilización de los lados del cuerpo de manera estable, dándose cuando no hay dominancia de uno de los lados sobre el otro.

La lateralidad, para su correcto desarrollo, ha de pasar por tres etapas (Ver Figura 6), recogidas por Ferré, Catalán, Casaprima y Mombiela (2000). 


\section{ETAPAS LATERALIDAD}

Prelateral: gracias al movimiento y el desarrollo sensorial, el niño va desarrollando esta etapa, en la que se van activando los hemisferios cerebrales, de modo que se vaya estableciendo la prelateralidad de manera adecuada. Esta etapa tendría una fase inicial monolateral alternante, seguida de otra de actividad duohemisférica, de transición a la siguiente etapa.

Lateral: se caracteriza por la resolución de las asimetrías, utilizando ambos brazos y piernas. Es capaz de coordinar un brazo con la pierna opuesta, automatizando adecuadamente patrones de movimiento. Esta etapa es muy importante porque se activa el cuerpo calloso y se activa la vía cruzada de movimiento.

Contralateral: se produce la correcta relación e intercambio de información entre los dos hemisferios, fruto de la activación anterior del cuerpo calloso. De este modo, cada hemisferio, aunque ambos participan en todas las acciones, tiene una especialización concreta.

Figura 6. Etapas de desarrollo de la lateralidad

\section{Creatividad, inteligencias múltiples y lateralidad}

No existen estudios que vinculen directamente los tres elementos, pero sí podemos encontrar ciertas relaciones entre estos. Pero respecto a la relación establecida entre las inteligencias múltiples y la creatividad, podemos decir que Gardner (1995) y Ferrando (2006), afirman que ambas no se pueden concebir por separado, destacando la existencia de una relación entre los tipos de creatividad y las distintas inteligencias. Asimismo, y haciendo alusión a la existencia en cada persona de las ocho inteligencias, y formas distintas de ser inteligente en cada una de ellas, cabe destacar que hay varias maneras de ser creativo en las mismas.

La relación que podemos establecer entre la lateralidad, las inteligencias múltiples y la creatividad va más en términos de alteraciones o dificultades en el aprendizaje, como destaca Mesonero (1994) ya que, cuando un niño tiene problemas de aprendizaje, por ejemplo con la lectura, puede deberse, entre otros factores, a las alteraciones de la lateralidad y a las dificultades para discriminar la derecha de la izquierda. Asimismo, Mayolas, Villarroya y Reverter (2010) establecen una relación entre la lateralidad y la motricidad ocular, con las repercusiones que esto tienen a nivel lector. Por último, estudios de García, Acuña y Argudín (1992), destacan la relación existente entre niños con lateralidad cruzada y menor rendimiento, por problemas con la memoria, orientación, motricidad, etc. Es decir, si existe una alteración en la lateralidad, esto va a repercutir en las diferentes inteligencias y en la creatividad, ya que no se van a poder desarrollar al máximo. Asimismo, se establece una relación más clara y directa entre los problemas de lateralidad y las inteligencias cinestésico-corporal y viso-espacial, por su vinculación con el movimiento, la orientación, los desplazamientos, etc.

\section{Método}

Partiendo de la relación que se establece de manera directa entre ambas temáticas, creatividad e inteligencias múltiples, junto con la lateralidad, se ha llevado a cabo una intervención en la que:

- Por una parte, se miden los niveles de cada una de las Inteligencias Múltiples, potenciando las más bajas, así como el tipo de lateralidad.

- Por otra parte, se lleva a cabo una intervención, fomentando la creatividad a través de las anteriores Inteligencias y el trabajo de la lateralidad. 
- Finalmente, para ver el efecto en los niveles de creatividad, se realiza el Test de Creatividad Infantil (TCl) a dos grupos, uno experimental, en el que se ha llevado a cabo la intervención, y otro control, para comparar ambos niveles.

Para ello contamos como objetivo: mejorar la creatividad a través del trabajo con la lateralidad y las inteligencias viso-espacial, Cinestésico-corporal y lingüística.

La investigación llevada a cabo es de tipo cuantitativa, y cuasiexperimental, ya que la muestra no ha podido ser aleatoria.

Las variables que se miden son tres: los niveles de inteligencias múltiples, la lateralidad y los niveles de creatividad tras una intervención planteada en base a los resultados obtenidos en las anteriores pruebas.

Los instrumentos de medida utilizados han sido los siguientes:

- Cuestionario del profesor para diagnosticar inteligencias múltiples en primaria, elaborado por Armstrong (2001). Consta de una serie de afirmaciones, a las que se contesta con "sí", "no" o "alguna vez", otorgando así una puntuación y pudiendo obtener el nivel en cada una de las inteligencias.

- Test de lateralidad de la prueba neuropsicológica, adaptado por Martín Lobo, $G^{a}$-Castellón, Rodríguez y Vallejo (2011). Consta de diez pruebas para comprobar la lateralidad del ojo, oído, mano y pie, siendo un total de 40 pruebas.

- Test de Creatividad Infantil (TCI) (2008), confeccionado por M. Romo Santos, V. Alfonso Benlliure y M. J. Sánchez-Ruiz. Se trata de un test de unos 45 minutos de duración, con posible aplicación individual o colectiva y de $1^{\circ}$ a $6^{\circ}$ de Educación Primaria.

Respecto a la fiabilidad de este test, cabe destacar, como así recogen sus autores, que en el análisis de la concordancia entre evaluadores, se encontró que la correlación intraclase para medidas promedio es de 0,95 , valor equiparable al alfa de Cronbach, y de 0,80 para medidas individuales.

La validez del mismo se comprobó con juicios de personal experto en dibujos infantiles. El nivel de acuerdo interjueces, evaluado mediante el coeficiente alfa de Cronbach, fue de 0,80 .

El test consta de dos fases bien diferenciadas, las cuales valoran aspectos diferentes. Estas fases son:

- La fase inicial, considerada como fase de creación del modelo o formulación del problema consiste en dar las instrucciones generales del test y llevar a cabo la realización de una composición con una serie de pegatinas dadas, pudiendo utilizar todas aquellas que se quiera, con la disposición y manipulación que cada uno desee.

- La segunda fase, llamada de realización del dibujo o solución del problema, consiste en que el niño elabore un dibujo partiendo de la composición que ha hecho previamente con las pegatinas, y pudiendo utilizar diversos materiales, los cuales también vienen determinados en el test.

Finalmente, se valoran diferentes ítems de ambas partes, obteniendo una puntuación final total, y correspondiéndose esta con unos percentiles. 
La realización del estudio se ha hecho en Segovia. La muestra de intervención (grupo experimental) se compone de 30 participantes, de los cuales 18 son chicos y 12 son chicas, cursando todos ellos $2^{\circ}$ de Educación Primaria, con una edad comprendida entre los 7 y los 8 años. A esta muestra, para el estudio comparativo, hay que añadirle otro grupo (el control), el cual tiene las mismas características, contando con 18 chicos y 12 chicas, del mismo colegio, curso y edad.

\section{Resultados y discusión}

Los resultados obtenidos en el cuestionario de Inteligencias Múltiples son los que a continuación se muestran, estando estos resumidos (Ver Tabla 3), y recogiendo los niveles del grupo entero.

\begin{tabular}{|lcccccccc|}
\hline \multicolumn{7}{|c|}{ Tabla 3. Niveles en cada una de las inteligencias } \\
\hline \multicolumn{7}{|c}{ I. Lingüística I. Matemática I. Viso-espacial I. Musical I. Cinestésico-corporal I.Interpersonal I. Intrapersonal I. Naturalista } \\
\hline Media & 5,73 & 7,02 & 5,50 & 7,13 & 5,28 & 6,87 & 6,82 & 7,03 \\
Moda & 6 & 9 & 7 & 7 & 7 & 7,5 & 7 & 8 \\
Mediana & 6 & 7 & 6 & 7 & 5,5 & 7 & 7 & 7,25 \\
Máximo & 9 & 9 & 9 & 10 & 10 & 10 & 9 & 10 \\
Mínimo & 1 & 3 & 0 & 3 & 0 & 3 & 2 & 3,5 \\
\hline
\end{tabular}

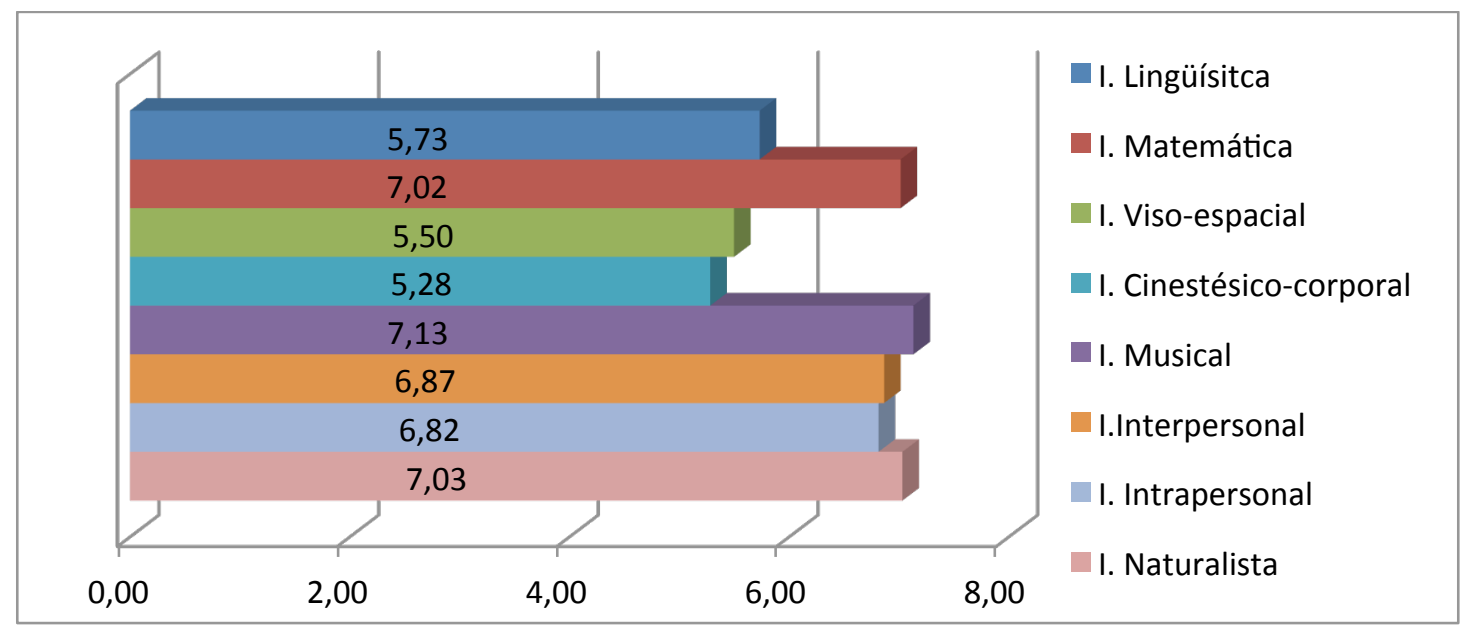

Figura 9. Media registrada en cada inteligencia

Los niveles de inteligencias múltiples varían de unas a otras, encontrando que aquellas en las que la puntuación es menor es en la Lingüística, la Viso-espacial y la Cinestésico-corporal. En estas tres, la media es cercana al 5, considerándose eso una puntuación media. Sin embargo, en el resto de inteligencias, las puntuaciones medias oscilan entre en 6,8 y el 7,13 , siendo consideradas medio-altas.

Por ello, a continuación vamos a hacer un desglose de aquellas que han registrado puntuaciones menores, de modo que podamos comprobar cuál es la frecuencia y el porcentaje en cada una de las puntuaciones. Para ello partimos de siguientes baremos, aportados por el Test de inteligencias múltiples realizado:

- Nivel bajo: puntuación de 0 a 2 .

- Nivel medio-bajo: puntuación de 2,5 a 4. 
- Nivel medio: puntuación de 4,5 a 6 .

- Nivel medio-alto: puntuación de 6,5 a 8 .

- Nivel alto: de 8,5 a 10.

\begin{tabular}{|ccc|}
\hline \multicolumn{4}{|c|}{ Tabla 4. Distribución de puntuaciones en la Inteligencia Lingüística } \\
\hline Nivel I. Lingüísitca & $\mathrm{N}^{0}$ de alumnos & Porcentaje \\
\hline Bajo & 3 & $10,00 \%$ \\
\hline Medio-bajo & 6 & $20,00 \%$ \\
\hline Medio & 10 & $33,33 \%$ \\
\hline Medio-alto & 6 & $20,00 \%$ \\
\hline Alto & 5 & $16,67 \%$ \\
\hline
\end{tabular}

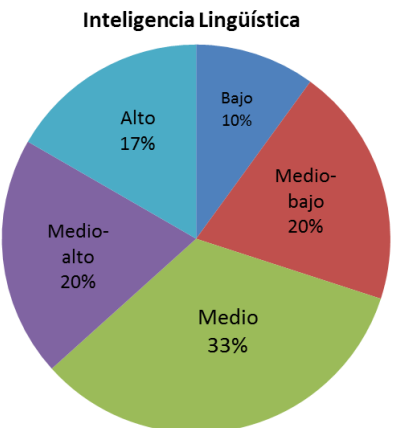

Figura 10. Porcentaje de puntuaciones-Inteligencia Lingüística

Como se puede observar, las puntuaciones altas y medio-altas no alcanzan a sumar el $50 \%$ de alumnos, siendo las más numerosas las puntuaciones medias, registradas por 10 niños de 30.

\begin{tabular}{|ccc|}
\hline \multicolumn{3}{|c|}{ Tabla 5. Distribución de puntuaciones en la Inteligencia Viso-espacial } \\
\hline Nivel I. Viso-espacial & $N^{0}$ de alumnos & Porcentaje \\
\hline Bajo & 3 & $10,00 \%$ \\
\hline Medio-bajo & 7 & $23,33 \%$ \\
\hline Medio & 7 & $23,33 \%$ \\
\hline Medio-alto & 10 & $33,33 \%$ \\
\hline Alto & 3 & $10,00 \%$ \\
\hline
\end{tabular}

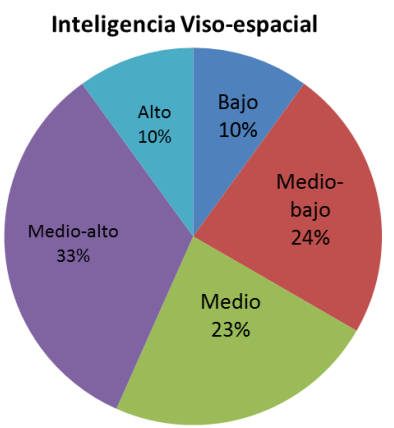

Figura 11. Porcentaje de puntuaciones-Inteligencia Viso-espacial

En este caso, el porcentaje mayor de puntuaciones corresponde a un nivel medio-alto. Pero, pese a ello, el sumatorio de este con el de puntuaciones altas, tampoco llega a ser el de la mitad de la muestra, sino que en esta, las puntuaciones dominantes son medias, medio-bajas y bajas.

\begin{tabular}{|lcc|}
\hline Tabla 6. Distribución de puntuaciones en la Inteligencia Cinestésico-corporal \\
\hline Nivel I. Cinestésico-corporal & $\mathbf{N}^{0}$ de alumnos & Porcentaje \\
\hline Bajo & 5 & $16,67 \%$ \\
\hline Medio-bajo & 4 & $13,33 \%$ \\
\hline Medio & 9 & $30,00 \%$ \\
\hline Medio-alto & 10 & $33,33 \%$ \\
\hline Alto & 2 & $6,67 \%$ \\
\hline
\end{tabular}

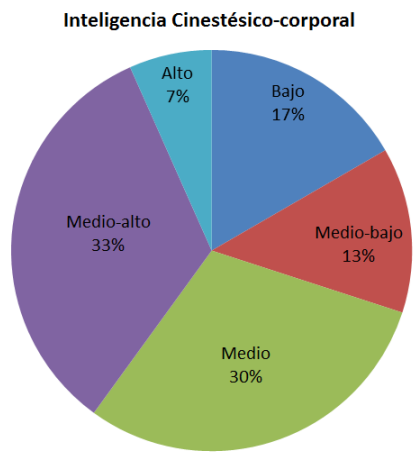

Figura 12. Porcentaje de puntuacionesInteligencia Cinestésico-Corporal

Las puntuaciones en esta inteligencia son muy similares a nivel de porcentaje entre las medio-altas y las medias, siendo ligeramente superior, por un alumno, en las primeras. 
Independientemente de esto, el sumatorio de porcentajes de las puntuaciones medias y las inferiores, es superior al $50 \%$, siendo esto lo dominante.

Las pruebas de lateralidad, adaptadas por Martín Lobo, Ga -Castellón, Rodríguez y Vallejo (2011), también han sido llevadas a cabo antes de la intervención, recogiendo los resultados, de manera resumida, en la Tabla 7, pudiendo obtener una visión global del estado de desarrollo de la lateralidad en dicho aula.

\begin{tabular}{|lcc|}
\hline \multicolumn{3}{|l|}{ Tabla 7. Tipos de lateralidad } \\
\hline Tipo de lateralidad & $N^{0}$ alumnos & Porcentaje \\
\hline Diestro & 26 & $86,67 \%$ \\
\hline Zurdo & 2 & $6,67 \%$ \\
\hline Lateralidad cruzada & 1 & $3,33 \%$ \\
\hline Lateralidad sin definir & 1 & $3,33 \%$ \\
\hline
\end{tabular}

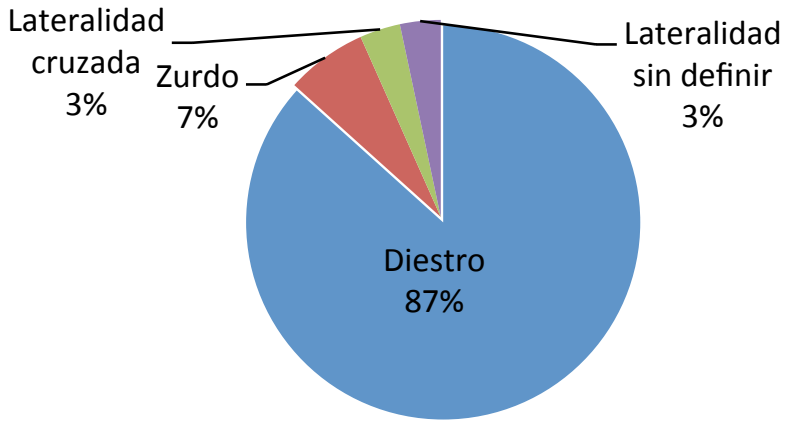

Figura 13. Porcentajes de puntuaciones: Tipos de lateralidad

Los resultados obtenidos en la realización de la prueba de la lateralidad han determinado que 26 alumnos $(86,67 \%)$ son diestros, no presentando ningún problema. Asimismo, en dicha muestra, encontramos dos alumnos zurdos (6,67\%), de los cuales hay que destacar que solo uno de ellos presenta complicaciones relacionadas con la zurdería, mientras que el otro no. Además, un alumno, $(3,33 \%)$, presenta una lateralidad cruzada, siendo diestro con cruce de pie. Finalmente, hay un alumno con lateralidad sin definir, obteniendo en las pruebas que es diestro de mano, zurdo de oído, mientras que en el ojo y en el pie no lo tiene definido.

La creatividad, como ya se ha comentado anteriormente, se ha medido con el Test de Creatividad Infantil $(\mathrm{TCl})$. Las otras pruebas fueron pasadas previas a la intervención, como base para lo que había que mejorar. Sin embargo, este ha sido realizado después de la intervención, de modo que podamos ver si esta ha tenido los resultados esperados en los niveles de creatividad.

Para ello, se ha llevado a cabo el test en el grupo experimental en el que se ha desarrollado y en otro equivalente (grupo control), pudiendo establecer una evaluación comparativa.

\begin{tabular}{|lc|}
\hline Tabla 8. Resultados del grupo experimental \\
\hline Valores \\
\hline Media & 86,53 \\
\hline Moda & 85 \\
\hline Mediana & 85 \\
\hline Mínimo & 65 \\
\hline Máximo & 99 \\
\hline
\end{tabular}

\begin{tabular}{|lc|}
\hline \multicolumn{2}{|l|}{ Tabla 9. Resultados del grupo control } \\
\hline Media & Valores \\
\hline Moda & 58,67 \\
\hline Mediana & 65 \\
\hline Mínimo & 65 \\
\hline Máximo & 20 \\
\hline
\end{tabular}


Como podemos observar en las Tablas 8 y 9, así como en la Figura 14 las diferencias que encontramos entre los dos grupos, respecto a la media aritmética, son significativas, siendo esta bastante más alta en el grupo experimental que en el grupo control. Asimismo, los niveles mínimos y máximo registrados en cada uno de los grupos, son bastante diferentes, sobre todo en lo relativo al mínimo.

Si tenemos en cuenta la clasificación aportada por los autores del test, dividiendo las puntuaciones en altas (por encima de 75), medias (entre 25 y 75) y bajas (menos de $25)$, podemos ver que en el grupo experimental, la media se encuentra dentro de las puntuaciones altas, mientras que en el grupo control es media. Asimismo, en el grupo experimental, el mínimo se encuentra dentro de las puntuaciones medias, mientras que en el control, se encuentran en las bajas.

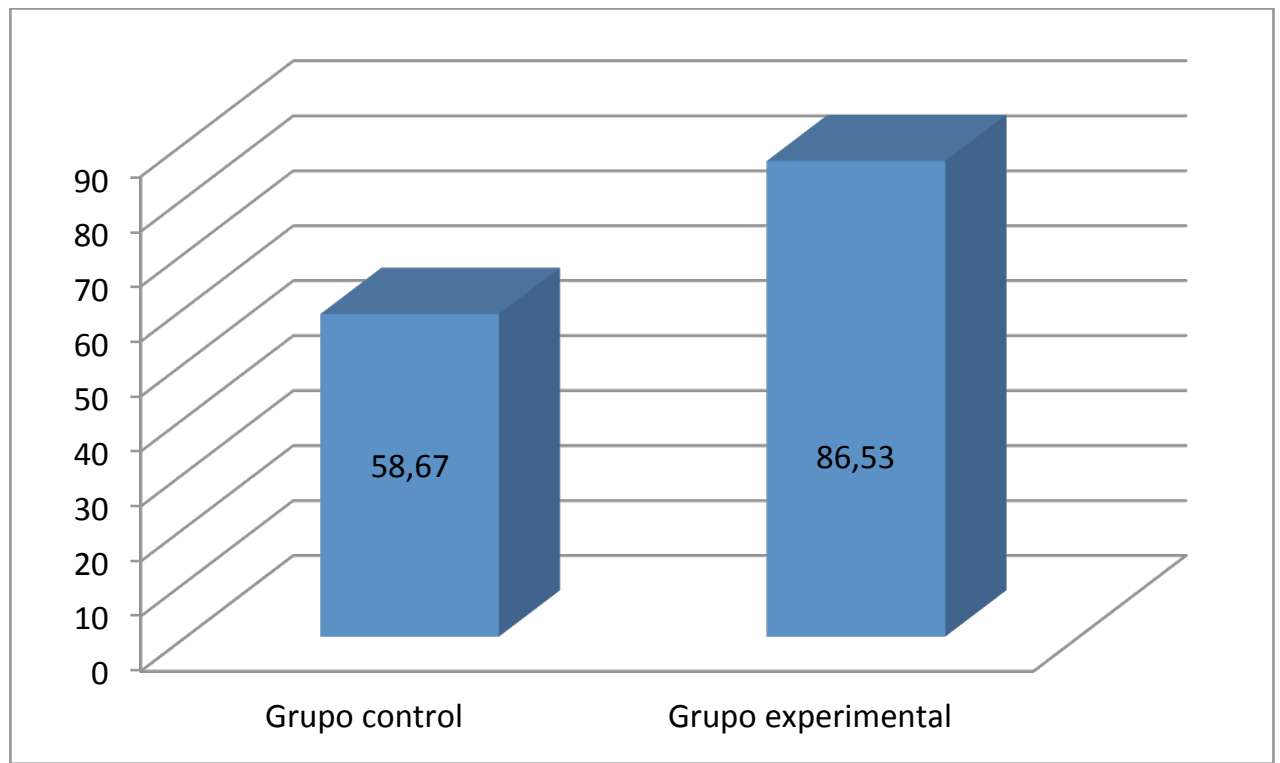

Figura 14. Media Test de Creatividad Infantil en el Grupo control y experimental

\section{Conclusiones}

Tras un análisis en profundidad de los resultados, podemos comprobar que las diferencias encontradas entre el grupo experimental con respecto al control son significativas. Esto pone de manifiesto la consecución de los objetivos planteados, consiguiendo desarrollar la capacidad creativa de los alumnos, así como llevar a cabo un trabajo de manera satisfactoria con diferentes agrupaciones y a través del trabajo de la lateralidad y las inteligencias Lingüística, Viso-Espacial y Cinestésico-Corporal.

Los niveles de creatividad del grupo experimental han sido superiores que las del control, quedando demostrada la eficacia de la intervención a través de las Inteligencias Múltiples y la lateralidad.

Se ha podido establecer una relación entre las alteraciones de la lateralidad (lateralidad cruzada, sin definir y uno de los zurdos) y puntuaciones bajas en las Inteligencias Cinestésico-corporal y Viso-espacial. Asimismo, se ha podido comprobar la necesidad que hay de trabajar la creatividad desde edades muy tempranas ya que, de no ser así, esta se ve disminuida con el paso del tiempo.

Respecto a la relación con las Inteligencias Lingüística, Viso-espacial y Cinestésicocorporal, podemos analizar como realizando actividades que engloben y fomenten el desarrollo de las mismas, se consiguen mejores resultados. La lateralidad, pese a que 
ha de estar establecida a los 7 años, hemos visto que algunos alumnos con 7-8 años aun no la tienen constituida, o presentan problemas de cruces laterales.

Asimismo, se puede ver cómo los problemas de lateralidad cruzada afectan al rendimiento académico general y a aspectos particulares relacionados con las inteligencias Cinestésico-corporal y Viso-espacial, pudiendo también haber problemas que relacionan las Inteligencias Múltiples y la creatividad con problemas de aprendizaje.

\section{Referencias}

Abad, S., Brusasca, M.C., y Labiano, L.M. (2009). Neuropsicología infantil y educación especial. Revista Intercontinental de Psicología y Educación, 11(1), 199-216.

Alexander, A.L. Hurley, S., Samsonov, A., Adluru, N., Hosseinbor, A., Mossahebi, P. Tromp. D., Zaksnewski, E. y Field, A. (2011). Characterization of Cerebral White Matter Properties Using Quantitative Magnetic Resonance Imaging Stains. Brain Connectivity, 1(6), 423-446.

Amabile, T.M. (1983). The Social Psychology of Creativity. New York: Springer-Verlag.

Amarís, M. (2002). Las múltiples inteligencias. Psicología desde el Caribe, 10, 27-38.

Arieti, S. (1976). Creativity: the Magic Synthesis. Nueva York: Basic.

Armstrong, T. (1999). Las inteligencias múltiples en el aula. Buenos Aires: Manantial.

Armstrong, T. (2001). Inteligencias múltiples: cómo descubrirlas y estimularlas en sus hijos. San José, Costa Rica: Editorial Norma.

Armstrong, T. (2008). Eres más listo de lo que crees. Barcelona: Ediciones Oniro.

Berembaum, S.A. (1998). Developmental Neuropsychology. Lawrence: Erlbaum Association.

Bernal, J. (2003). Música y creatividad, en Bernal, J., y Gallardo, M.A. (2009). Medición de la creatividad en la educación musical escolar. Revista Internacional de Creatividad aplicada total, 11, 2-13.

Binet, A. (1894). Introduction a la Psychologie Experimental. París: Alcan.

Binet, A., \& Simón, T.H. (1905). Methodes nouvelles pour le disagnotic du niveau intellectuel des anormaux. L'année Psychologique, 11, 191-244.

Bullmore, E., \& Sporns, O. (2009). Complex Brain Networks: Graph Theoretical Analysis or Structural and Functional Systems. Neuroscience, 10, 186-198.

Carlsson, I., Wendt, P.E., \& Risberg, J. (2000). On the Neurobiology of Creativity. Differences in Frontal Activity Between High and Low Creative Subjects. Neuropshychologia, 38, 873-885.

Castillo, J. (2002). Las Inteligencias Múltiples en el mejoramiento de la calidad. En Guzmán, B. y Castro, S. (2006). Las inteligencias múltiples en el aula de clases. Revista de investigación, 58, 177-210.

Davidson, R.J., Jackson, D.C., \& Kalin, N.H. (2000). Emotion, Plasticity, Context and Regulation: Perspectives from Affective Neuroscience. Psychological Bulletin, 126, 890-906.

De Bono, E. (1970). El pensamiento lateral. Manual de creatividad. Buenos Aires: Paidós.

De Bono, E. (1994). El pensamiento creativo. El poder del pensamiento lateral para la creación de nuevas ideas. Buenos Aires: Paidós.

De la Torre, S. (1991). Evaluación de la creatividad: TAEC, un instrumento de apoyo a la Reforma. Madrid : Escuela Española. 
De Prado, D. (2001). Educrea(te): Enseña-Aprende a ser creativo. Santiago de Compostela: Meobook.

Desrosiers, P. (2005). Psicomotricidad en el aula. Barcelona: INDE Publicaciones.

Dorsch, D. (1985). Diccionario de Psicología. Barcelona: Herder.

Escobar, A. y Gómez, B. (2006). Creatividad y función cerebral. Revista Mexicana de Neurociencia, 7(5), 391-399.

Esquivias, S. (2004). Creatividad: definiciones, antecedentes y aportaciones. Revista Digital Universitaria, 5(1), 1-17.

Ferrando, M. (2006). Creatividad e inteligencia emocional: un estudio empírico en alumnos con altas capacidades. (Tesis doctoral). Universidad de Murcia, Murcia.

Ferré, J., Catalán, J., Casaprima, V., Mombiela, J. (2000). El desarrollo de la lateralidad infantil. Niño diestro- Niño zurdo, $2^{\circ}$ ed. Barcelona: Lebón.

Ferré, J., y Aribau, E. (2002). El desarrollo neurofuncional del niño y sus trastornos. Barcelona: Lebón.

Fink, A., Grabner, R.H., \& Benedek, M. (2009). The Creative Brain: Investigation of Brain Activity during Creative Problem Solving by Means of EEG and fMRI. Human Brain Mapping, 30, 734-48.

Flaherty, A.W. (2005). Frontotemporal and Dopaminergic Control of Idea Generation and Creative Drive. The Journal of Comparative Neurology, 493, 147-153.

Gándara, J. (2007). Neurobiología del arte. Un modelo de autoestimulación visual creativa. Revista de Psiquiatría.com, $11(4)$.

García, E. (2008). Neuropsicología y Educación. De las neuronas espejo a la teoría de la mente. Revista de psicología y educación, 1(3), 69-90.

García, M., Acuña, M. y Argudín, O. (1992). Algunas consideraciones sobre la lateralidad cruzada y aprendizaje en niños. Revista del Hospital Psiquiátrico de la Habana, 33(2), 171-177.

Gardner, H. (1983). Estructura de la mente. La teoría de las Inteligencias Múltiples. México: FCE.

Gardner, H. (1993). Inteligencias múltiples: la teoría en la práctica. Barcelona: Paidós.

Gardner, H. (1995). Inteligencias múltiples: la teoría en la práctica. Barcelona: Paidós.

Gardner, H. (1999). La educación de la mente y el conocimiento de las disciplinas. Barcelona: Paidós.

Gardner, H. (2001). La inteligencia reformulada, las inteligencias múltiples en el siglo XXI. Barcelona: Paidós.

Chávez, R.A., Graff-Guerrero, A., García-Reyna, J.C., Vaugier, V., Cruz-Fuentes, C. (2004). Neurobiología de la creatividad: resultados preliminares de un estudio de activación cerebral. Salud Mental, 27(3), 38-46.

Greicius, M. (2008). Resting-state Functional Connectivity in Neuropsychiatric Disorders. Curr Opin Neurol., 21, 424430 .

Guilford, J.P. (1951). Guilford Test for Creativity. Beverly Hills, California: Sheridan Supply Company.

Guilford, J. P. (1965). Psicología General. México: Diana.

Guilford, J. P. (1967). The Nature of Human Intelligence. Nueva York: McGraw-Hill.

Guilford, J. P. (1986). La naturaleza de la inteligencia humana. Barcelona: Paidós.

Jiménez, J., Velázquez, J.C. y Jiménez, P. (2003). Psicomotricidad, cuentos y juegos programados. Madrid: La Tierra Hoy.

Le Boulch, J. (1981). El desarrollo psicomotor desde el nacimiento hasta los 6 años. Madrid: Doñate. 
Le Boulch, J. (1987). La educación psicomotriz en la escuela primaria. Buenos Aires: Paidós.

El Norte de Cartilla (10 de septiembre de 2013). Los 13.782 alumnos de Infantil, Primaria y Educación Especial de Segovia comienzan el curso con normalidad. El Norte de Castilla. http://www.elnortedecastilla.es/20130910/local/segovia/alumnos-infantil-primaria-educacion201309101428.htm

Mackinnon, D. W. (1980). Naturaleza y cultura del talento creativo: herencia y medio. Madrid: Narcea.

Martín Lobo, P., García-Castellón, C., Rodríguez, I. y Vallejo, C. (2011). Test de lateralidad de las pruebas neuropsicológicas. Instituto de Neuropsicología y Educación. Madrid: Fomento.

Martínez, J.M. y Rimm, S. (1985). Cuestionarios de creatividad. Madrid: San Pio X.

Mayolas, M.C., Villarroya, A. y Reverte, J. (2010). Relación entre la lateralidad y los aprendizajes escolares. Revista Apuntes de Educación Física y Deportes, 101, 32-42.

Mesonero, A. (1994). Psicología de la educación psicomotriz. Universidad de Oviedo: Ediuno.

Mosston, M y Ashworth, S (1996). La enseñanza en la Educación Física. La reforma de los estilos de enseñanza. Barcelona: Hispano-Europea.

Ortiz, C.P. (2004). Cuadernos de Psicobiología Social 1. Introducción a una Psicobiología del Hombre. Lima: Fondo Editoral de la UNMSM.

Ortiz, M. (2008). Biomechanics of Traumatic Brain Injury. Computer Methods in Applied Mechanics and Engineering, 197(51), 6692-4701.

Osborn, A. F. (1957). Applied Imagination: Principles and Procedures of Creative Thinking (Revised edition). Nueva York: Charles Scribner's Sons.

Pérez, P.M. (2006). Creatividad e innovación: una destreza adquirible. Revista Teoría de la Educación, 21(1), 179-198.

Pérez, P. (2009). Creatividad e innovación. Una destreza adquirible. Salamanca: Ediciones Universidad de Salamanca.

Piaget, J. (1969). El nacimiento de la inteligencia del niño. Madrid: Aguilar.

Poincaré, H. (1913). Science et méthode. París: Flammarion.

Portellano, J.A. (1989). La disgrafía. Madrid: CEPE.

Portellano, J.A. (2005). Introducción a la neuropsicología. Madrid: McGraw-Hill.

Renzulli, J.S., Smith, L.H., White, A.J., Callahan, C.M., Hartman, R.K. y Westberg, K.L. (2001). SCRBSS. Escalas para la valoración de las características de comportamiento de los estudiantes superiores. Salamanca: Amarú Ediciones.

Rigal, R. (2006). Educación motriz y educación psicomotriz en Preescolar y Primaria. Zaragoza: INDE Publicaciones.

Rius, M.D. (1989). Enciclopedia del desarrollo de los procesos grafomotores. Madrid: Seco Olea Ediciones.

Robinson, K. (2009). El elemento. Barcelona: Grijalbo.

Romo, M., Alfonso, V. y Sánchez, M.J. (2008). Test de Creatividad Infantil. Madrid: TEA.

Shaffer, D. y Kipp, K. (2007). Psicología del desarrollo. Infancia y adolescencia. México: Thomson.

Spearman, C. (1923). The Nature of Intelligence and the Principles of Cognition. Londres: MacMillan.

Sternberg, R. (1985). Más allá del Cl: Una teoría triárquica de la inteligencia humana. Nueva York: Cambridge University Press.

Torrance, E.P. (1962). Guiding Creative Talent. Englewood Cliffs, NJ: Prentice Hall. 
Torrance, E.P. (1974). The Torrance Test of Creative Thinking. Norms Technical. Manual Research Edition. Princeton $\mathrm{NJ}$ : Personnel Press.

Tuttle, L. (1980). Ideas for identification of Gifted Children. Illinois: Marion.

Wallach, M.A., \& Kogan, N. (1965). Modes of Thinking in Young Children. A Study of the Creativity-Inteligence Distintion. New York: Holt, Rinehart y Winston, Inc.

Wallas, G. (1926). The Arts of Thought. Nueva York: Harcourt-Brace.

Welch, L. (1946). Recombination of Ideas in Creative Thinking. Journal of Applied Psychology, 30, 638-643. 